\title{
Medicina Comportamental del SuEÑo: UN CAMPO EMERGENTE DENTRO DE LA PSICOLOGÍA DE LA SALUD Y LA MEDICINA DEL SUEÑO
}

\author{
Hernán Andrés Marin Agudelo, \\ Laboratorio del sueño y neurociencias cognitivas; Grupo de Neurociencias de Antioquia, Universidad de Antioquia, Medellin, Colombia; \\ Grupo de Medicina Comportamental del Sueño, Universidad Cooperativa de Colombia, Envigado, Colombia; Corporación Universitaria Lasallista, Caldas, Colombia. \\ Francisco Lopera, \\ Laboratorio del sueño y neurociencias cognitivas, Grupo de Neurociencias de Antioquia, Universidad de Antioquia, Medellin, Colombia
}

\begin{abstract}
Behavioral sleep medicine is a subspecialty both of sleep medicine and clinical and health psychology that addresses the explanation of cognitive-behavioral focuses that are relevant to identify contributing psychological factors in the development and/or maintenance of sleep disorders. Apart from being a specialty in the design of empirically validated cognitive-behavioral assessment and non pharmacologic intervention techniques for the whole spectrum of sleep disorders, behavioral sleep medicine develops strategies to identify aspects related to life quality in terms of health and sleep disorders, as well as the relationship between stress and sleep. Plenty of researches have been carried out using this approach and many disciplines have contributed to its action field configuration, of interest both for psychologists and health practitioners in general.
\end{abstract}

Keywords: Behavioral sleep medicine, cognitive-behavioral models, intervention techniques, clinical formulation.

\section{RESUMEN}

La medicina comportamental del sueño, es una subespecialización de la medicina del sueño y la psicología clínica y de la salud, encargada específicamente de la explicación de focos (cognitivo-comportamentales), importantes en la identificación de los factores psicológicos que contribuyen al desarrollo y/o el mantenimiento de los trastornos del sueño. Además de ser una especialización en el desarrollo de técnicas de evaluación e intervención cognitivocomportamentales empírico-validadas y otras medidas interventivas de corte no farmacológico para el espectro entero de los trastornos del sueño, se ocupa del desarrollo de estrategias que permitan identificar los aspectos relacionados con la calidad de vida en salud y las patologías del sueño, y de la relación entre estrés y sueño. Muchas investigaciones se han desarrollado desde esta óptica, lo mismo que muchas disciplinas han aportado para la configuración de este campo de acción, el cual atañe tanto a psicólogos como a otros profesionales de la salud en general.

Palabras Clave: Medicina comportamental del sueño, Modelos cognitivos comportamentales, técnicas de intervención, formulación clínica.

La historia de la medicina del sueño es relativamente corta, sus inicios se identifican a principios del siglo XX, específicamente en la década de los treinta, y está marcada por un alto desarrollo tecnológico el cual permitió un acercamiento más

Fecha recepción: 12/03/2007 • Fecha aceptación: 09/06/2007

Dirección de Correspondencia:

Calle 62 No.52-59, Sede Administrativa Universitaria SIU,

Torre 1, Zona Asistencial, Laboratorio del Sueño,

Medellín - Colombia

E-mail: behavioralsleepmedicine@yahoo.com.ar objetivo a su estudio, a diferencia de las concepciones filosóficas que existían hasta la fecha (Dement, 2000; Dement \& Vaughan, 2000; Shepard et al., 2005). La psicología había tenido también muchos acercamientos, tanto desde la descripción, como desde la intervención en los trastornos del sueño, incluso mucho antes de la aparición de la medicina del sueño la psicología estuvo encargada de dichos procesos, los cuales fueron avanzando hasta formalizar, en la primera década del siglo XXI, un área de especialización denominada Medicina Comportamental del Sueño (Stepanski \& Perlis,

Este producto fue patrocinado por Colciencias y la Universidad de Antioquia, con la financiación del Proyecto: "Somnolencia excesiva, hábitos de sueño y accidentalidad en conductores de servicio público urbano e interdepartamental”. Código: 1115-345-18974. 
2000, 2003). La denominación y aparición de programas académicos formales en Medicina Comportamental del Sueño es reciente, llegando al cubrimiento de propuestas doctorales y posdoctorales y libros especializados. En Latinoamérica el posicionamiento de esta subespecialidad, ha sido lento, sin embargo, existen programas de intervención en trastornos de sueño y grupos investigativos estructurados, aunque este desarrollo reciente, trae consigo muchas preguntas y cuestionamientos sobre el avance de esta especialización de la medicina del sueño y la psicología clínica (Stepanski 2003).

El objetivo central de este artículo es mostrar un panorama de este campo ocupacional, tanto para los psicólogos, como neurólogos y profesionales de la salud en general, que aspiren o quieran dedicarse al mismo, propiciando una discusión que apoye los fundamentos conceptuales aquí descritos.

\section{DESARROLLO CONCEPTUAL E HISTÓRICO}

La Medicina Comportamental del Sueño (MCS), surge recientemente como un área de especialización dentro del campo más amplio de la medicina del sueño y la psicología clínica y de la salud. Los primeros aportes conceptuales y las técnicas de tratamiento, se plantearon en los años 30, y en la actualidad se a tenido avances significativos que, más allá del tratamiento del insomnio, incluyen el tratamiento de los trastornos por somnolencia excesiva diurna, trastornos del sueño en la infancia, trastornos del ritmo circadiano y parasomnias (Stepanski \& Perlis, 2000, 2003). Formalmente, los aspectos conceptuales de la medicina comportamental del sueño comprenden aquellas dimensiones del comportamiento que favorecen o no el sueño, los mecanismos normales y anormales del sueño, la prevención de los mismos, la relación que tienen éstos con la calidad de vida del sujeto y el tratamiento de los trastornos del sueño y los problemas comportamentales y emocionales asociados.

El término "Medicina Comportamental del Sueño", se desarrolló gracias a las discusiones entre Michael Perlis y Edward J. Stepanski, en la asociación americana de medicina del sueño, en los últimos años de la década de los noventa. El primer uso oficial del nombre ocurrió en 2000, cuando Dan Buysse, como presidente de la Academia Americana de Medicina del Sueño, formó un comité presidencial sobre MCS, encargado especialmente de cuatro funciones específicas: a) desarrollar un mecanismo para la acreditación de los profesionales en MCS, b) desarrollar las pautas para los programas de formación profesional en MCS, c) desarrollar programas educativos sobre MCS para la presentación en las diferentes reuniones profesionales, y d) establecer tarifas para los servicios de MCS, en los Estados Unidos (Shepard et al., 2005). El comité desarrolló el primer examen de certificación para MCS en el 2003.

Formalmente, la medicina Comportamental del sueño (MCS), se refiere a la rama de la medicina del sueño y de la psicología clínicas de la salud orientada a:
1) Explicación de focos (cognitivo-comportamentales), importantes en la identificación de los factores psicológicos que contribuyen al desarrollo y/o el mantenimiento de los trastornos del sueño.

2) Especialización en el desarrollo y el abastecimiento de técnicas de evaluación e intervención cognitivocomportamentales, empírico-validadas, y/u otras medidas interventivas de corte no farmacológico para el espectro de los trastornos del sueño.

3) Desarrollo de estrategias que permitan identificar los aspectos de la calidad de vida relacionada con la salud y las patologías del sueño, y la relación entre estrés y sueño.

Estos puntos argumentan la revisión que se viene haciendo desde esta perspectiva en relación al sueño, sus trastornos y la salud (Marín, 2006, 2007).

La medicina del sueño ha sido un campo multidisciplinario desde su inicio, con aportes prominentes de la psiquiatría, la neurología, la neumología, la otorrinolaringología, la pediatría y la psicología. Las prácticas de MCS existieron antes de la era de la medicina moderna del sueño, pues los problemas del sueño estaban en gran parte bajo el cuidado de psicólogos y de psiquiatras, los cuales utilizaron métodos que actualmente recuerdan los principios de entrenamiento en higiene del sueño (Shepard et al., 2005). Desde la psicología podríamos definir algunos puntos teóricos en la consolidación de la medicina comportamental del sueño, como el modelo comportamental del sueño de Webb (1988), el modelo de insomnio de Morin, (1998), el modelo cognitivo de Harvey (2002); el modelo comportamental de la somnolencia excesiva diurna de Marín y Vinaccia (2004b). Dichos modelos se convierten en la base para la intervención y, desde la evaluación, existen aportes psicométricos y formulaciones clínicas para una intervención relevante (Lichstein \& Nau, 2003).

Son muchas las disciplinas que confluyen en la aparición de la medicina comportamental del sueño (ver figura 1), tanto a nivel explicativo, como interventivo, generando un campo disciplinar específico.

Dentro del planteamiento conceptual de la medicina del sueño, al analizar las bases teóricas de la misma, queda claro su argumento multidisciplinario, como se observa en la interacción de diferentes áreas tanto de la medicina del sueño, como de la psicología clínica, para cumplir con sus principios de formular, abastecer y evaluar los diferentes aspectos de la calidad de vida en los pacientes con trastornos del sueño.

\section{LOS PRIMEROS ANTECEDENTES Y EL GRAN ÉNFASIS EN EL INSOMNIO}

Los primeros antecedentes en MCS se centran en Laird, investigador de la Universidad de Colgate, quien escribió sobre 


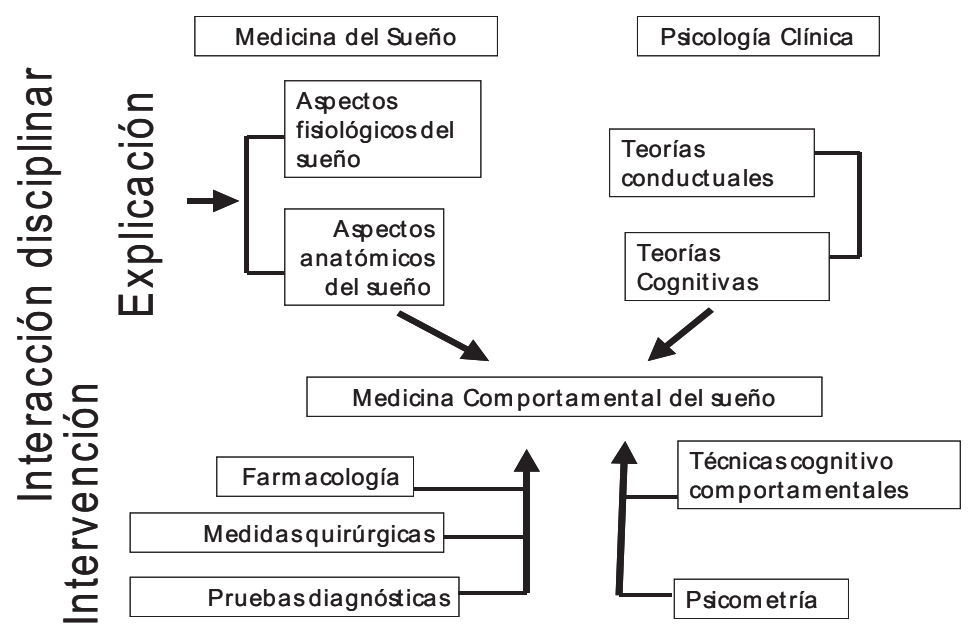

Figura1. Interacción de los diferentes modelos teóricos que fundamentan la medicina comportamental del sueño. Como se observa en la figura, la interacción de las diferentes áreas tanto de la medicina del sueño, como de la psicología clinica, han apuntado a describir, el argumento teórico de la medicina comportamental del sueño. Lasflechas indican como los diferentes aportes disciplinares, confluyen hacia la explicación del nuevo campo emergente.

cómo el sueño inadecuado afectaba excesivamente a los trabajadores por turnos ocupacionales, los efectos del sueño corto en el aprendizaje, y la necesidad del sueño en los niños, así como sobre muchos otros asuntos durante los años 20 (Stepanski \& Perlis, 2000, 2003). Otro antecedente significativo es el trabajo bien conocido de Jacobson sobre insomnio, el cual se puede citar como el principio de la MCS. Jacobson desarrolló los principios y técnicas de la relajación muscular progresiva (técnica que hoy en día es una de las estrategias de elección en el tratamiento del insomnio), basado en su trabajo con pacientes que padecían de problemas somáticos relacionados con tensión (Jacobson, 1938). Además, hipotetizó que el aumento del despertar contribuye a un número amplio de quejas médicas comunes (Jacobson, 1934). El desarrollo de la terapia de relajación de Jacobson, aplicada al insomnio, proporcionó una descripción exacta de lo qué eventualmente sería llamado "insomnio psicofisiológico", concepción que describiría en el libro "Usted puede dormir bien". Aunque a Jacobson se le recuerda más, por desarrollar dichas técnicas de relajación muscular progresiva, en sus escritos también demostró una comprensión profunda del insomnio. Su descripción de los factores comportamentales y cognitivos, asociados al insomnio, es absolutamente similar a nuestra comprensión actual de estas dimensiones, al indicar cómo los componentes culturales y emocionales en interacción con todos los aspectos de la sociedad moderna, generan bastante activación, conduciendo al aumento del despertar nocturno y alternativamente al insomnio (Jacobson, 1934).

Otros antecedentes de la MCS, con respecto a las investigaciones realizadas para el insomnio, tenían como objetivo el entender los avances producidos para explicar los mecanismos subyacentes del mismo en los años 60 . Gran parte de ésta investigación se desarrolló en la Universidad de Chicago bajo la dirección de Allan Rechtschaffen. En la misma década, Monroe, Hauri,
Robinson y Zimmerman, demostraron, en diferentes trabajos, cómo el despertar fisiológico y cognitivo, estaba relacionado con pacientes con mal dormir (Hauri, 1968; Monroe, 1967; Stepanski \& Perlis, 2000, 2003). El papel del arousal fisiológico y cognitivo, adquiría cada vez más importancia entre los factores que perpetuaban e influían en la manifestación del insomnio crónico, recibiendo una comprobación empírica (Stepanski \& Perlis, 2000, 2003).

El trabajo de Jacobson, Monroe, Hauri, Robinson y Zimmerman, direccionó los estudios empíricos de los tratamientos para el insomnio, basados en la relajación, durante los años 70 y principios de los años 80 . Posteriormente, Hauri publicó estudios que demostraban la eficacia del biofeedback en el tratamiento del insomnio a principio de los 80 (Stepanski y Perlis, 2000, 2003). Esta investigación era particularmente notable porque la eficacia diferencial del tratamiento, dependió de las características y el tipo de tratamiento realizado en los pacientes. Por ejemplo, el despertar creciente, medido por los niveles de electromiografía (EMG), pero no con biofeedback de electroencefalografía (EEG), predijo un mejor resultado del tratamiento.

Estas contribuciones del biofeedback, hoy en día constituyen tratamientos probablemente eficaces para el insomnio crónico en 1999, dentro de la práctica de la Asociación Americana de Medicina del Sueño. Sobre los últimos 15 años, las terapias de relajación se han incorporado gradualmente o han sido substituidas por otros acercamientos y programas cognitivocomportamentales y multi-componentes (TCC) (Stepanski \& Perlis, 2000, 2003). El biofeedback, parece haber caído de peso, en cuanto a ser un tratamiento primario de elección para el insomnio y se utiliza poco. Otra causa para esto, es probablemente la naturaleza intensiva en tiempo de este tratamiento, con 15-62 sesiones de una hora de entrenamiento requeridas para el tratamiento, en comparación con las 7-12 sesiones de tratamiento con programas multicomponentes. 
Dentro de la aplicación de las técnicas de condicionamiento operante y clásico, sumadas a las técnicas cognitivas para el tratamiento del insomnio, se encontraban la técnica de control del estímulo y la restricción del sueño. Los tratamientos que investigaban la eficacia de la terapia del control del estímulo para el insomnio crónico tuvieron un renacimiento (hay que recordar que antes de basarse en la teoría, se realizaban de manera empírica), en los años 70, con la aplicación de los principios de la teoría del comportamiento, aplicados al problema del insomnio. Bootzin en 1972, utilizó conceptos de la teoría del aprendizaje para crear la terapia del control del estímulo (TCE) para el insomnio (Stepanski \& Perlis, 2000, 2003). Este acercamiento innovador se basó en los principios del condicionamiento operante y señaló los comportamientos desadaptativos relacionados con el sueño, y el tiempo despierto en cama, como blancos terapéuticos (Lacks 1987; Lacks, Bertelson, Surgeman, \& Kunkel, 1983; Stepanski \& Perlis, 2000, 2003). Esta técnica, continúa siendo uno de los tratamientos más utilizados para el insomnio, y se incluye en programas multicomponentes de tratamiento. Otro de los componentes de los programas de intervención es el entrenamiento en higiene del sueño. Este acercamiento es extremadamente popular, y se recomienda casi universalmente para el tratamiento del insomnio. Las reglas consideradas se han desarrollado durante años, aunque hay pocos estudios de estas reglas como tratamiento "independiente", y parece ser considerado un componente necesario, pero no suficiente, en el tratamiento del insomnio (Stepanski \& Perlis, 2000, 2003).

En segundo lugar los acercamientos cognitivos, estuvieron marcados en principio por los principios de la intención paradójica en la formulación de un tratamiento para el insomnio, indicando a los pacientes permanecer despiertos tanto como sea posible, después de irse a la cama en la noche (Stepanski \& Perlis, 2000, 2003). Muchos estudios han comprobado la eficacia de esta técnica en el tratamiento en el insomnio, sin embargo, la investigación sobre la misma, como tratamiento de elección para el insomnio, ha desaparecido de la literatura, y no parece ser utilizada comúnmente en la actualidad.

En los años 80, otro de los aportes de la MCS para los programas de intervención multicomponente, estuvo marcado con la aparición de la terapia de la restricción del sueño (TRS), desarrollada por Spielmann, Saskin y Thorpy (1987). Esta técnica comportamental buscaba reducir sistemáticamente tiempo en la cama, para aumentar el impulso homeostático del sueño y posteriormente aumentar el tiempo de eficacia de sueño en la cama. Este aporte de intervención técnica se ha convertido en un tratamiento extensamente usado, y se incluye rutinariamente como parte de los programas de tratamiento para el insomnio. Otra contribución importante de Spielmann, en ese tiempo, fue un modelo teórico para entender el desarrollo del insomnio crónico y en cierta forma conceptualizar los trastornos del sueño desde la MCS, acercándose cada vez más a la visión del paciente a nivel individual. El modelo, se conoce como historia natural del insomnio (Spielmann, Caruso, \& Glovinsky, 1987), y según el mismo existen tres tipos de factores actuantes en el insomnio: a) los factores que predisponen, o rasgos de resistencia que reducen el umbral necesario para desencadenar el insomnio, b) las circunstancias que lo precipitan, o factores temporales y otros que rodean el inicio del insomnio, y c) los factores que perpetúan, o las variables que contribuyen al manejo del insomnio a lo largo del tiempo. Otro modelo que aparecería en los noventa en la comprensión de la explicación de la patología insomne, es conocido como el modelo del microanálisis del insomnio (Morin, 1998), el cual describe aspectos tales como la hiperactivación o múltiples estímulos elevan el nivel de activación por encima del umbral crítico interrumpiendo la secuencia natural de relajación, somnolencia e inicio del sueño, las repuestas más típicas al insomnio las cuales incluyen preocupación por el número de horas de sueño y pensamientos reiterativos sobre los efectos residuales del día, la tensión muscular, la inquietud física general, y las consecuencias del día siguiente, que implican fatiga, alteración del estado de ánimo, irritabilidad, malestar social y disminución del rendimiento. El tercer modelo explicativo del insomnio, aparece en el 2002 con Harvey, quien retoma algunos aspectos centrales del componente cognitivo y de activación del modelo microanalítico del insomnio de Morin, y los modelos cognitivos del procesamiento de la información de Beck. El modelo explica el papel de la acción cognitiva, en la generación sintomática de aspectos que mantienen el insomnio, definiendo la acción de las creencias cognitivas en cuatro aspectos: 1) la excesiva negatividad en el tono cognitivo, 2) un nivel de arousal alto y distrés, 3) Una atención selectiva y monitoreo de conductas elevado, y 4) una percepción distorsionada del déficit de sueño. Estos cuatro factores generan tanto las conductas desadaptativas, que refuerzan las creencias en la noche sobre la percepción negativa de déficit de sueño real, y durante el día la percepción errada del funcionamiento diurno. Cabe anotar que esta misma perspectiva, es la que explica, al retroalimentarse noche y día, el proceso de mantenimiento del insomnio (Harvey, 2002).

En general, hasta ahora la medicina comportamental del sueño se había centrado en el abordaje del insomnio, empezando por la modificación de conducta de aquellos factores implicados en el inicio mantenimiento y duración del sueño; posteriormente al surgir los modelos explicativos del insomnio, empiezan a surgir los programas de intervención multicomponente, retomando cada uno de los mismos y centrándose en focos específicos para el abordaje de estos trastornos del sueño.

\section{EL SURGIMIENTO DE LA INTERVENCIÓN DE LA SOMNOLENCIA EXCESIVA DIURNA, EL OTRO APORTE DE LA HISTORIA}

La intervención de la somnolencia excesiva diurna, aparecería como un punto aislado hasta ahora para la medicina comportamental del sueño. Esto es importante, porque solo hasta las décadas de los 70 y 80 , surgirían acuerdos puntuales en las descripciones y clasificaciones en general de los trastornos del sueño, quedando claro algunos aspectos, dentro de los cuales se destacan la aparición de las clasificaciones 

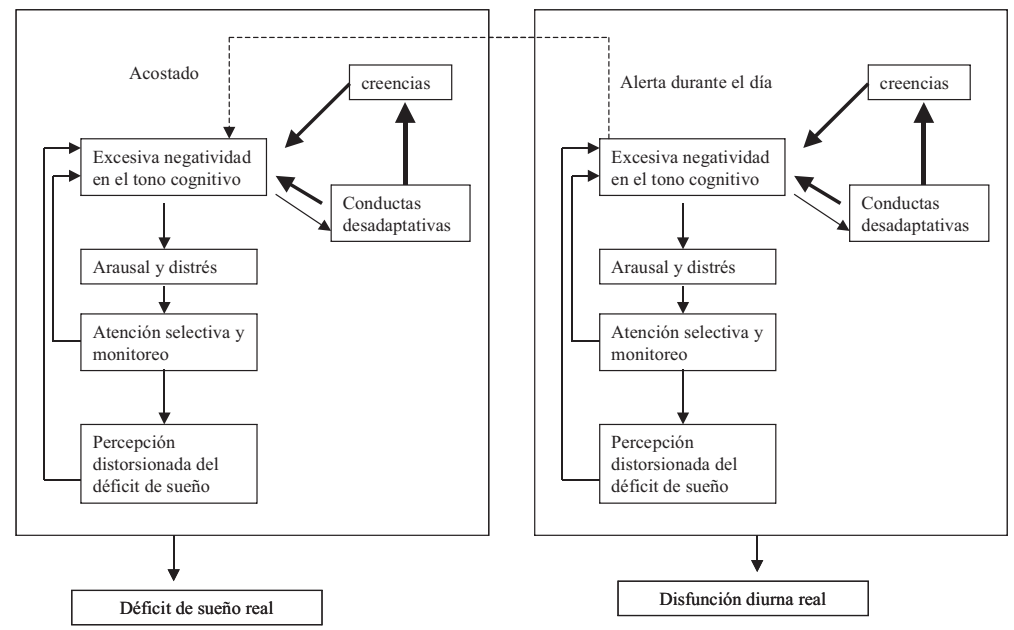

Figura 2. Modelo cognitivo del insomnio (Harvey, 2002), en donde se explica, como los factores cognitivos, modulan el mantenimiento delinsomnio.

diagnósticas que especificarían, aún más, lo criterios para el diagnóstico de los desordenes caracterizados por somnolencia excesiva diurna, bajo la mirada de la fisiología y la medicina el sueño, mientras que el aporte comportamental en la descripción de estos síndromes seguía sin aparecer concretamente, pese a algunos puntos relevantes en los componentes sintomáticos comportamentales de los mismos (como la frecuencia de la conducta y la intensidad de la misma, entre otros). Esto comenzaría a cambiar sucesivamente desde principios de la década de los 70 hasta principios de los 80 , debido a varios acontecimientos, entre los que se encuentra la aparición de la primera escala comportamental de medición de la somnolencia excesiva diurna a cargo de Hodes, Dement y Zarcone (1973), conocida como la escala de somnolencia Stanford (medición del alerta y la somnolencia en varios momentos del día).

Aparecería además, en la década de los ochenta, la descripción del test de latencia múltiple, desarrollado por Caskardon y Dement en 1987, que empezaba a abordar la manifestación en un tiempo determinado de menos de 10 minutos, de un componente conductual de la somnolencia, como es la tendencia a quedarse dormido, en varios espacios de tiempo en el día. Otros hechos que colaboraron en la búsqueda de estrategias puntuales en la intervención de la somnolencia excesiva diurna, es la aparición y la explicación del déficit de sueño acumulado y las influencias de este en la presencia de la somnolencia excesiva diurna, como fruto del análisis de los diferentes modelos de regulación del sueño (Dement \& Vaughan, 2000; Dinges 1989).

Utilizando estos mecanismos de regulación de sueño y basándose en las teorías comportamentales, Manber, Bootzin, Acebo y Caskardon en 1996, plantearían las bases para la técnica comportamental de regulación de sueño en la somnolencia excesiva diurna. Esta técnica encontraría sus antecedentes en las reflexiones de Dinges, en la década de los 80 , además de otros planteamientos teóricos y técnicos para la intervención de la narcolepsia desarrollados por Hauri en 1982. Otras técnicas comportamentales utilizadas en la intervención de la somnolencia excesiva diurna, es la restricción del sueño de 4 a 5 horas, durante una semana (Dinges et al., 1997), y los efectos de planificación de la siesta (Akerstedt 1985; Akerstedt, Hume, Minors, \& Waterhouse, 1993; Broughton 1988; Buysse, Brawman, Monk, \& Reynutds, 1992; Dinges 1995; Kolko, 1984; Monk, Buysse, Carrier, Billy, \& Rose, 2001). Otros aportes específicos en cuanto a los trastornos respiratorios del sueño o desordenes respiratorios del sueño, son en primer lugar la adherencia al tratamiento (Haynes, 2005), en segundo lugar la intervención condicionada para corregir el ronquido posicional (Cartwright, Lloyd, Lily, \& Kravitz, 1985, Cartwright, Ristanovic, Diaz, Calderelli, \& Alder, 1991) y por último la intervención con desensibilización sistemática para pacientes con claustrofobia (Edinger \& Radtke, 1993), lo mismo que la comprensión teórica de la narcolepsia y la intervención comportamental de la misma (Garma \& Marchand 1994; Marín \& Vinaccia, 2005b). En la década de los 90, surgieron varias escalas de medición para la somnolencia excesiva diurna, lo mismo que diferentes subescalas en pruebas completas para los trastornos del sueño, como son la escala de somnolencia Epworth (Johns, 1991); igualmente existieron otros aportes importantes en el estudio de la prevalencia de la somnolencia excesiva diurna (Jonson, Breslau, Roth, Roers, \& Rosenthal, 1999, Weaver 2001), como el desarrollo del concepto de somnificidad (Johns, 2002) y somnolencia activa y pasiva (Kim \& Young, 2005).

Estos aspectos colaboraron para la aparición del modelo comportamental de la somnolencia excesiva diurna (Marín \& Vinaccia, 2004a, Marín \& Vinaccia, 2005a), el cual concibe la somnolencia, como una respuesta subjetiva caracterizada por un deseo irresistible de dormir en circunstancias inapropiadas o no deseadas, modulada por tres sistemas conductuales en la estructuración de la misma: 1) variables situacionales que alteran o fragmentan el sueño nocturno, determinadas por 
aquellas conductas que causan privación de sueño y que van moduladas en consonancia con el modelo de regulación de sueño y factores circadianos del mismo, 2) sistemas de creencias o pensamientos que fragmentan el sueño, basados en cogniciones y evaluaciones negativas sobre la eficacia y necesidad de sueño, además de cogniciones que favorecen la conducta activa y el estado de alerta mas allá de los patrones circadianos y de regulación de sueño del individuo, y 3) características fisiológicas que fragmentan el sueño nocturno, algunas patogénesis de tipos específicos de hipersomnias (déficit de hipocretina en la narcolepsia, características morfofisiológicas en la apnea obstructiva de sueño), otras referidas a la alteración en sistemas de respuesta fisiológicos de manifestación conductual que están directamente relacionados con los síntomas dentro de los cuales se encuentran la arquitectura de sueño y ciertas variables relacionadas con la calidad de sueño como la latencia de sueño (considerada como la medida de sueño en la cual una persona tarda en dormirse), la duración de sueño (que hace referencia a la cantidad de horas que el sujeto es capaz de mantenerse dormido) y la eficiencia habitual de sueño (que hace referencia a la valoración de porcentaje de sueño que un sujeto puede estar dormido, sobre el total de tiempo que permanece acostado sin dormirse, multiplicado por cien) (Marín \& Vinaccia 2004b). Estas variables se modulan en interacción con otras que fragmentan el sueño, como una tendencia más fuerte a desarrollar somnolencia excesiva diurna. Los factores antes mencionados, describen aspectos centrales que en general producen privación de sueño y la manifestación conductual de sueño excesivo ante situaciones pasivas y activas, con una caracterización de curso de acuerdo a la clasificación internacional de trastornos del sueño, de significancia clínica, leve, moderada y grave (Marín \& Vinaccia, 2004a, Marín \& Vinaccia, 2005a).

Este modelo se amplia para explicar las manifestaciones del comportamiento sintomático en el día, dando respuesta a la operacionalización de la manifestación sintomática
(Caskardon \& Dement, 1987, Mitler, 1990, 2000). La manifestación sintomática comportamental de la somnolencia excesiva explicada por el modelo (Marín \& Vinaccia, 2005b), plantea que la respuesta de somnolencia excesiva diurna esta definida como la manifestación de la frecuencia diurna de una latencia de sueño inferior a diez minutos manifestada en varios episodios sucesivos de sueño diurno entre las 9:00 a.m. y las 6:00 p.m. (se plantea la intermitencia de la misma, en los periodos de las 9:00 a.m. a las 12:30 a.m., y de las 3:00 p.m. a las 6:00 p.m.), además de la cantidad de tiempo de sueño diurno (característica que se presenta además del sueño nocturno). Esta frecuencia está delimitada exponencialmente, por la tendencia circadiana de la frecuencia de estas conductas (Monk, 1991), manifestándose en la somnificidad o manifestación conductual del sueño diurno, ante situaciones pasivas que exigen poca respuesta motivacional o arousal de activación cognitiva, o activas que exigen un nivel mayor de arousal, o activación. Estas concepciones se aprecian en las figuras 3 y 4 .

En ambas figuras se destaca la interacción de los componentes en la manifestación sintomática de la somnolencia, componentes que retoman los modelos de regulación del sueño, sumados a las diferentes manifestaciones sintomáticas que han descrito varios autores, dentro del test de Latencia Múltiple, la escala de Somnolencia Epworth, la escala de somnolencia de Karolinska, entre otras, y define la interacción de estas respuestas en la manifestación multicomponente de la somnolencia excesiva diurna. Este modelo permite tener una visión objetiva para la formulación clínica, al definir las conductas objetivo de la modificación conductual para reducir el impacto del sueño diurno, utilizando los procesos fisiológicos implicados en la regulación del sueño, como el condicionamiento del proceso $\mathrm{S}$ mediante siestas planificadas, el nivel máximo del alerta para ubicar las actividades que exigen un mayor compromiso (Akerstedt., 1993, Manber et al., 1996), o la utilización de la cronoterapia o condicionamiento del proceso S, desplazando dicha tendencia (Akesterd, 1985).

\section{RSmn $=f($ LS + TTSD + CESD $) x$ TC \pm Smnf}

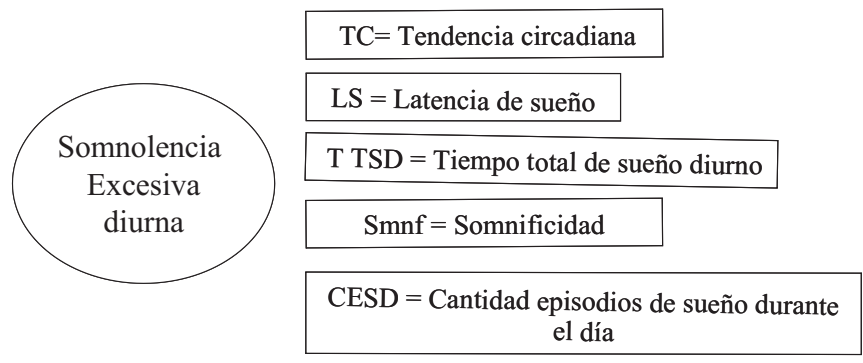

Figura 3. Modelo objetivo de la somnolencia excesiva diurna (Marin y Vinaccia, 2005a). Ecuación de la somnolencia excesiva diurna. La respuesta de somnolencia excesiva (RSmn), es igual a la suma de los factores: latencia de sueño (LS), tiempo total de sueño diurno (TTSD), la cantidad de episodios de sueño durante el día (CESD), por la tendencia circadiana de estos aspectosy más o menos la respuesta de somnificidad (Smnf). Esta ecuación amplía la visión de la ecuación comportamental del sueño de Webb 1988 y Akerstedty Folkard 1990. 


\section{Antecedentes fisiológicos o cognitivo-conductuales}

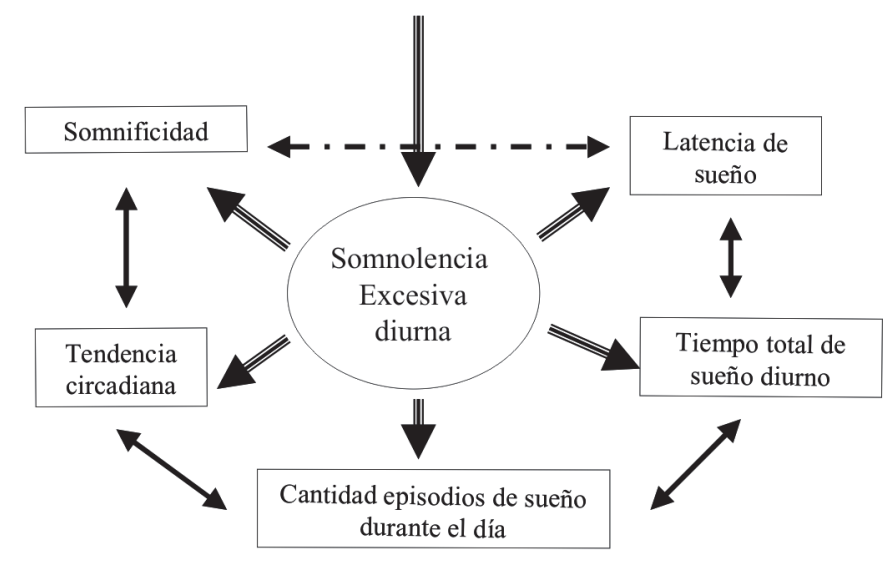

Figura 4. Manifestación sintomática de la somnolencia excesiva diurna, (Marín y Vinaccia, 2005a). Como se aprecia, se destaca la interacción de los componentes en la manifestación sintomática de la somnolencia,componentes que retoman tanto los modelos de regulación del sueño (en los procesos circadianos y la interacción de los componentes de sueño, en la respuesta de la somnolencia), sumados a las diferentes manifestaciones sintomáticas que han descrito varios autores, dentro del test de Latencia Múltiple, la escala de Somnolencia Epworth, la escala de somnolencia de Karolinska, entre otras, y define la interacción de estas respuestas en la manifestación multicomponente de las somnolencia excesiva diurna.

\section{LOS APORTES EN LAS PARASOMNIAS, LOS TRASTORNOS DEL RITMO CIRCADIANO Y LA CALIDAD DE VIDA}

Otros aportes de la MCS se centran en el abordaje de las parasomnias, tales como las pesadillas (Cavior \& Deutsch, 1975; Germain \& Nelson, 2003; Halliday, 1987; Miller \& DiPilato, 1983; Reid, Ahmed, \& Levie, 1981; Pellicier, 1993), planteando la utilización de la desensibilización sistemática, en el abordaje de las mismas, y la técnica de los sueños lúcidos e imágenes (Zadra \& Phil, 1997, Spoodmarker y Bout, 2006) o la intervención, incluso en el sonambulismo, a través de la hipnosis (Hauri, Silber, \& Boeve, 2004; Howsam, 1999; Hurwitz, Mahowald, Schenck, Schluter, \& Bundlie, 1991). También se ha indicado el biofeedback en la intervención en enuresis (Doleys, 1977, Longstaffe, Moffatt, \& Whalen, 2000). En cuanto a los trastornos del ritmo circadiano, avances como la fototerapia (Campbell,1995, Czeisler \& Dijk, 1995, Terman, Terman, \& Hall, 2000), la utilización de diversas técnicas comportamentales en el tratamiento del trastorno de fase atrasada de sueño (Lack \& Wrigth., 2007), la cronoterapia o tratamiento que busca sincronizar los ritmos biológicos (Akerstedt \& Gillberg, 1981), o las técnicas de adherencia al tratamiento y suspensión de fármacos tanto en el insomnio, como en la somnolencia excesiva diurna, lo mismo que el desarrollo de conceptos sobre la somnolencia excesiva diurna y la explicación cognitivo-comportamental de algunos trastornos del sueño, constituyen los aportes realizados la primera década del siglo XXI, además del surgimiento de la revista especializada Behavioral sleep medicine y el libro Treating Sleep disorders: Principles and Practice of behavioral sleep medicine, que se han convertido junto con otros textos escritos en ingles y en español, como puntos de confluencia y de divulgación teórica e investigativa de este campo de acción.

Otro campo amplio, es el tratamiento de los trastornos del sueño en la infancia, para los cuales se pide ayuda en los centros de terapia del comportamiento (Kuhnn \& Elliot, 2003) y que incluyen desde problemas para conciliar o mantener el sueño, hasta miedo a la noche y a una amplia gama de parasomnias, tales como terrores nocturnos o somnambulismo. Las interrupciones de las rutinas normales del sueño, pueden tener efectos serios en el bienestar tanto de los niños, como de los padres, y se han ligado al funcionamiento cognoscitivo de los niños y a la regulación emocional, así como a la depresión, así como al desarrollo de otros problemas del comportamiento. Existen tratamientos disponibles, objeto de estudio de lo que se ha denominado también medicina pediátrica comportamental del sueño (Kuhnn \& Elliot, 2003). Los programas de intervención de los trastornos del sueño en lactantes y primera infancia, son explicados desde la terapia con enfoque comportamental, mientras que con niños mayores y adolescentes, quienes cuentan con actitudes más favorables a la comunicación y la persuasión, las intervenciones cognitivo-conductuales pueden ser los tratamientos de elección luego de una formulación clínica. Sin embargo, las intervenciones con enfoques comportamentales en la infancia, implican un componente cognitivo para trabajar con los padres, puesto que se tienen que alterar los pensamientos, las actitudes, y la creencias de los mismos, antes de que estén dispuestos y convencidos para emprender un programa de tratamiento comportamental (Kuhnn \& Elliot, 2003).

Otros acercamientos psicológicos para tratar los trastornos del sueño en la infancia, tales como las terapias estratégicas y la hipnosis, han sido divulgados en algunos estudios, pero su eficacia queda en duda. Es importante anotar que en el espectro de los trastornos del sueño en la infancia es donde la MCS, luego del insomnio, ha trabajado con mucha eficacia (Kuhnn \& Elliot, 2003).

En conclusión la MCS se ha ido posicionando lentamente como un campo de investigación atractivo para los psicólogos 
y los profesionales de la salud en general. Su desarrollo, estuvo en principio marcado, por los aportes y modelos explicativos del insomnio a nivel molecular, los aportes molares son de aparición reciente, lo mismo que el abordaje y la intervención de la somnolencia excesiva diurna. Existen diferentes aportes desde la intervención de las parasomnias y los trastornos del ritmo circadiano, lo mismo que en los trastornos del sueño en la infancia como áreas importantes que poco a poco se han ido desarrollando.

\section{PERSPECTIVAS FUTURAS}

La visión académica en la MCS así como la apertura de la misma en nuestro contexto, requiere que los especialistas adquieran conocimiento tanto de los conceptos claves en medicina del sueño como de la medicina comportamental, convirtiéndose quizás en el primer desafío hacia el futuro (Pigeon \& Crabtree, 2006; Stepanski \& Perlis, 2000, 2003). Desafortunadamente, casi no hay programas de entrenamiento formales en nuestro medio, y los pocos que existen se encuentran en Estados Unidos y en España. En el nivel pregraduado, existen pocos programas que apoyen, al menos, las bases conceptuales y prácticas sobre los aspectos clínicos y de la investigación en el sueño (Pigeon \& Crabtree, 2006; Stepanski \& Perlis, 2000, 2003). En Latinoamérica específicamente, el área de la MCS es una disciplina emergente con pocos profesionales capacitados para este fin; al mismo tiempo tanto las investigaciones en este campo como la producción bibliográfica a nivel internacional en ingles y en español es muy poca, solo existe una revista de medicina comportamental del sueño y un libro especializado en este campo. En Iberoamérica, España, Brasil y Colombia son los países que más han estado trabajando en la materia, tanto a nivel investigativo, con el aporte de Brasil y Colombia, como a nivel formativo e investigativo en España, en donde existen grupos de investigación con publicaciones internacionales y posicionados. Queda de antemano claro el desafío de generar en Latinoamérica programas de formación centrados en la MCS y en crear más grupos y asociaciones conformadas de investigadores (Marín \& Vinaccia, 2005a; Pigeon \& Crabtree, 2006).

Quizá el segundo desafío en la práctica de la MCS sea tomar una postura proactiva, y preguntarse qué factores representan una barrera al uso extenso de las modalidades de tratamiento cognitivo comportamental para los trastornos del sueño. Se espera que con un aumento en la investigación clínica, estos aspectos ganaran credibilidad como terapias de elección en la intervención y aportarán oportunamente a los códigos de diagnóstico y a la medicina del sueño en general. La MCS no es todavía el procedimiento estándar en la práctica clínica, las intervenciones comportamentales parecen ser más costosas, en cuanto a tiempo y dinero (Marín \& Vinaccia, 2005a; Pigeon \& Crabtree, 2006) y hay una carencia de conocimiento entre los profesionales dedicados a la medicina del sueño, sobre los procedimientos y la eficacia de la medicina comportamental en general, y sobre lo que es la MCS en detalle. Por otra parte, hay pocos clínicos en América del Norte y en Latinoamérica, que se interesen, investiguen y que se especialicen en la ofertar de servicios de MCS. Por último, la mayoría de las compañías de seguros y de los planes obligatorios de salud, no proporcionan la cobertura para el tratamiento de los trastornos del sueño desde esta óptica; y los que lo hacen, dejan de cubrir aspectos importantes de la intervención, reduciendo muchas veces el tipo de ésta (Marín \& Vinaccia, 2005a; Pigeon \& Crabtree, 2006).

En resumen, hay una carencia comprensible de conocimiento entre la comunidad médica, en cuanto a la importancia y eficacia del tratamiento cognitivo-comportamental en los trastornos del sueño. La falta de especialistas formados y disponibles en esta área, se debe, en gran parte, a que hay pocas oportunidades de entrenamiento en MCS, solo 10 programas de especialización y ocho con internado clínico en Estados Unidos, y alrededor de 4 programas posgraduados en España y recientemente uno en Colombia y, más aún, un pequeño e indeterminado número de sitios que proporcionan formación clínica en esta especialidad.

Los últimos 30 años han arrojado resultados muy importantes en la comprensión de los factores cognoscitivos y comportamentales que contribuyen a potencializar, perpetuar o mantener los trastornos del sueño en el adulto y en los niños. La alta eficacia de la terapia cognitivo-comportamental para el insomnio, constituye un avance significativo; sin embargo, dicho avance es un poco lento en los demás trastornos del sueño. Cada área descrita en la historia de la MCS todavía tiene muchos desafíos, aunque se estén desarrollando en este momento investigaciones que demuestran la eficacia de la intervención con MCS.

La MCS se encuentra en un período del crecimiento excepcional, con una ampliación de sus intereses, los cuales van más allá del insomnio, para incluir la somnolencia excesiva diurna, las parasomnias y los trastornos del ritmo circadiano. Un reto en el protocolo de tratamiento del insomnio, será el de establecer la eficacia de la terapia cognitivo-comportamental, en pacientes con insomnio transitorio y en pacientes con insomnio secundario. Una mejor comprensión de los mecanismos del insomnio a través de estos subtipos de pacientes podría conducir al diseño de estrategias más eficaces para adaptarlas al insomnio crónico, en realzar más la eficacia del tratamiento y en reducir el tiempo requerido para el mismo. Otro tópico en esta dirección, sería investigar qué determina que la terapia cognitivo-comportamental se pueda combinar con el tratamiento farmacológico. El abordaje de las limitaciones y los acercamientos de tratamientos combinados, pueden apoyar mejor la eficacia de los mismos y presentarlos como tratamientos de elección, en contraposición a las estrategias actuales, las cuales se basan en un tratamiento cognitivo-comportamental o farmacológico, como único protocolo de elección. 


\section{REFERENCIAS}

Akerstedt, T. (1985). Adjustment of physiological circadian rhythmsand the sleep-wake cycle to shift work. En S. Folkard, \& T. Monk (eds.), Hours of Work: Temporal Factors in Work Scheduling (pp. 185-198). New York: John Wiley \& Sons.

Akersted, T., \& Gillberg, M. (1981) The circadian variation of experimentally displaced sleep. Sleep, 4, 159-169.

Akerstedt, T., Hume, K., Minors, D., \& Waterhouse, J. (1993). Regulation of sleep and Naps on an irregular Schedule. Sleep, 16, 736-743.

Bootzin, R. (1972) Stimulus control treatment for insomnia. Proceedings of the 80 th annual convention of the America psychological association, 7, 395-396.

Broughton, R. J. (1988). SNC controlled circadian arousal and the afternoon "Nap Zone". Journal Sleep Research on Line, 1, 166-178.

Buysse, D. J ., Brawman, K. E., Monk, T. H., \& Reynutds, C.F. (1992). Napping and 24 hour sleep/wake patterns in healthy elderly and young adults. American Geriatrics Society, 40, 779-786.

Campbell, S. S. (1995). Effects of timed bright-light exposure on shift-work adaptation in middle-aged subjects. Sleep, 18, 408-416.

Carskadon, M. A., \& Dement, W. C. (1987). Daytime sleepiness: quantification of behavioral state. Neuroscience and Biobehavioral Reviews, 11, 307-317.

Cartwright, R., Lloyd, S., Lily J., \& Kravitz, H. (1985) Sleep position training as treatment for sleep apnea syndrome: a preliminary study. Sleep, 8, 87-94.

Cartwright, R., Ristanovic, R., Díaz, F., Calderelli, D., Alder, G. (1991) A comparative study of treatments for positional sleep apnea. Sleep 14, 546-52.

Cavior N, \& Deutsch A M. (1975) Systematic desensitization to reduce dream-induced anxiety. Journal of Nervous and Mental Disorders, 161, 433-435.

Czeisler, C. A., \& Dijk, D. J. (1995). Use of bright light to treat maladaptation to night shift work and circadian rhythm sleep disorders. Journal of Sleep Research, 4, 70-73.

Dement, W.C. (2000). History of sleep physiology and medicine. En: M. Kryger, T. Roth, \& W. Dement (eds.), Principles and Practice of Sleep Medicine, (3 ed., pp. 377-390). Philadelphia: W.B. Saunders.

Dement, W. C., \& Vaughan, C. (2000). Dormir Bien. Buenos Aires: Atlántida.

Dinges, D. F., Pack, F., Williams, K., Gillen, K. A., Powell, J. W., Ott, G. E., Aptowicz, C., \& Pack, A. I. (1997). Cumulative sleepiness, mood disturbance, and psychomotor vigilance performance decrements during a week of sleep restricted to 4-5 hours per night. Sleep, 20, 267-77.

Dinges, D. (1995). Napping strategies. Fatigue Symposium Proceedings (pp. 47-51). Washington, D.C.: NTSB and NASA Ames Research Center, Nov. 1-2.

Dinges, D. F. (1989). The nature of sleepiness: causes, contexts and consequences. En A. Stunkard, \& A. Baum (Eds.): Perspectives in Behavioral Medicine: Eating, Sleeping, and Sex (pp. 147-179). Hillsdale: Lawrence Erlbaum.
Doleys, D. M. (1977) Behavioral treatments, for nocturnal enuresis in children: a review of recent literature, Psichology bulletin, 84, 30-54.

Edinger J. D., \& Radtke, R A. (1993) Use of in vivo desensitization to treat a patient's claustrophobic response to nasal CPAP. Sleep, 16, 678-680.

Garma L., \& Marchand, F. (1994) Non-pharmacological approaches to the treatment of narcolepsy. Sleep,. 17(Suppl), S97-102.

Germain A, Nielson TA. (2003) Impact of imagery rehearsal treatment on distressing dreams, psychological distress, and sleep parameters in nightmare patients. Behavioral Sleep Medicine, 1, 140-154.

Hauri P. (1968). Effects of evening activity on early night sleep. Psychophysiology, 4, 266-77.

Hauri, P. J., Silber, M. H., \& Boeve, B. F. (2004) Treating parasomnias with hypnosis. Sleep, 27 (Supplemet), A286.

Halliday, G. (1987). Direct Psichological therapies for nightmares: a review. Clinicalpsychology review, 7, 501-523.

Harvey, A. G. (2002) A Cognitive model of insomnia. Behaviour research and therapy, 40, 869-893.

Haynes, P. L. (2005) The role of behavioral sleep medicine in the assessment and treatment of sleep disordered breathing. Clinical Psychology Review, 25, 673-705.

Hoddes, E., Dement, W. C., \& Zarcone, V. (1972). The development and use of the Stanford Sleepiness Scale (SSS). Psychophysiology, 9, 72.

Howsam, D. G. (1999) Hypnosis in the treatment of insomnia, nightmares, and night terrors. Australian Society of Hypnosis 27,32-39.

Hurwitz, T. D, Mahowald, M. W, Schenck, C. H, Schluter, J. L, \& Bundlie, S. R. (1991) A retrospective outcome study and review of hypnosis as treatment of adults with sleepwalking and sleep terror. Journal of Nervous and Mental Disorders, 179, 228-233.

Jacobson, E. (1934). You can sleep well. New York. Mac Graw Hill Boock Company.

Jacobson, E. (1938). Progressive relaxation. Chicago. University of Chicago press.

Johns, M. W. (1991). A new method for measuring day time sleepiness: the Epworth Sleepiness Scale. Journal of Sleep Resources, 14, 540-545.

Johns, M. (2002) Sleep propensity varies with behaviour and the situation in which it is measured: the concept of somnificity. Journal of Sleep Research, 11, 61-67.

Johnson, E. O., Breslau, N., Roth, T., Roers, T., \& Rosenthal, L. (1999) Psychometric evaluation of daytime Sleepiness and nocturnal Sleep onset scales in a representative community Sample. Biological psychiatry, 45, 764-770.

Kim, H., \& Young, T. (2005) Subjective daytime sleepiness: dimensions and correlates in the general population. Sleep, 28, 627- 636 .

Kolko, D. J. (1984) Behavioral treatment of excessive daytime sleepiness in an elderly woman with multiple medical problems. Journal of Behavioral Therapy \& Experimental Psychiatry,. 15, 341-345. 
Kuhnn, B.R., \& Elliot, A. J. (2003) Treatment efficacy in behavioral pediatric sleep medicine. Journal of psychosomatic research. 54, 587-597.

Lack, L., \& Wright, H (2007) Clinical Management of delayed phase disorder. Behavioral Sleep Medicine, 5, 57-76.

Lacks, P. (1987) Behavioral treatment for persistent insomnia, New York: Pergamonnd Presss.

Lacks, P., Bertelson, A.D., Surgeman, J., \& Kunkel, J. (1983) He treatment of sleep maintenance insomnia with stimulus-control techniques Behavioral resarch and therapy, 21, 291-295.

Lichstein, K., \& Nau, S. (2003) behavioral-cognitive science: the foundation of behavioral sleep medicine. En M. L. Perlis, \& K. L. Lichstein (eds), Treating Sleep Disorders: Principles and Practice of Behavioral Sleep Medicine (pp. 169-189). New York: Wiley.

Longstaffe, S., Moffatt, M., \& Whalen, J. (2000) A Randomized, Controlled Trial Behavioral and SelfConcept Changes After Six Months of Enuresis Treatment Pediatrics, 105, 935-940.

Manber, R., Bootzin R., Acebo, C., \& Carskadon, M. (1996) Behavioral treatments and sleepiness. The effects of regularizaring sleep-wake schedules on daytime sleepiness. Sleep, 19, 432-441.

Marín, H. A., \& Vinaccia, S. (2004a). Evaluación y tratamiento de la somnolencia excesiva diurna: una revisión. Psicología y Salud. 14, 245-255.

Marín, H A, \& Vinaccia, S. (2004b). Calidad de vida y somnolencia excesiva diurna. En L. Oblitas (ed.), Manual de psicología de la salud y hospitalaria. Libro electrónico recuperado de www.psicologiacientífica.com.

Marín, H.A., \& Vinaccia, S. (2005a). Contribuciones desde la medicina comportamental del sueño, al manejo de la somnolencia excesiva diurna. Psicología desde el caribe. $14,95-116$

Marín, H. A., \& Vinaccia, S. (2005b). Modelo CognitivoComportamental del síndrome de narcolepsia, cataplejía: Exposición teórica. Revista de sicopatología y Psicología Clínica, $10,153-172$.

Marín, H. A. (2006) Trastornos del sueño, Salud y Calidad de vida, En L. Oblitas, (ed.) Atlas de Psicología de la Salud. México: Psicom Editores.

Marín, H. A. (2007) Trastornos del sueño y Calidad de vida. Avances de la medicina del sueño en Latinoamérica. 1, 15-23.

Miller, W. R., \& DiPilato, M. (1983) Treatment of nightmares via relaxation and desensitization: A controlled evaluation. Journal of Consulting \& Clinical Psychology, 51, 870-877.

Mitler, M. M. (1990). Evaluación de la somnolencia excesiva. En G. Buela-Casal, \& J. F. Navarro (Eds.), Avances en la investigación del sueño y sus trastornos (pp. 241-258). Madrid: Siglo XXI.

Monk, T. H., Buysse, D. J., Carrier, J., Billy, B. D., \& Rose, L. R. (2001). Effects of afternoon "siesta" naps on sleep, alterness, performance, and circadian rhythms in the elderly. Sleep, 24, 680-687.
Morín, C. M. (1998) Insomnio, Asistencia y tratamiento psicológico. Madrid: Ariel Psicología.

Monroe, L. J. (1967). Psychological and physiological differences between good and poor sleepers. Journal of Abnormal Psychology, 72, 255-64.

Pellicier, X. (1993) Eye movement desensitization treatment of a child's nightmares: a case report. Journal of Behavioral Therapy \& Experimental Psychiatry, 24, 73-75.

Pigeon,W., \& Crabtree, V. (2006) The Future of Behavioral Sleep Medicine. American Academy of Sleep Medicine's Behavioral Sleep Medicine Committee, pp. 1-20.

Reid, W. H., Ahmed, I., \& Levie, C. A. (1981). Treatment of Sleepwalking: a controlled study. American Journal of Psychotherapy, 35, 27-37.

Shepard, J., Buysse, D., Cheeson. A., Dement, W., Goldberg, R., Guilleminault, C., et al. (2005) History of the development of sleep medicine in the United States. Journal of clinical Sleep Medicine, 1, 61-82.

Spielman, A. J., Saskyn, P., \& Thorphy, M. (1987) Treatment of chronic insomnia by restriction of time in bed. Sleep, 10 , 45-56.

Spielman, A, Caruso, L., \& Glovinsky, P. (1987) A behavioral perspective in insomnia treatment. Psychiatric Clinics of North America, 10, 541 - 553.

Spoormaker, V., \& Bout, J (2006) Lucid Dreaming Treatment for Nightmares: A Pilot Study. Psychotherapy \& Psychosomatic, 75, 389-394.

Stepanski, E. J. (2003) Behavioral sleep medicine: a historical perspective. Behavioral Sleep Medicine, 1, 4-21.

Stepanski, E. J., \& Perlis, M. L. (2000) Behavioral sleep medicine. An emerging subspecialty in health psychology and sleep medicine. Journal of Psychosomatic Resource, 49, 343347.

Stepanski, E. J., \& Perlis, M. L. (2003) Introduction of Behavioral sleep medicine. A historical perspective and commentary on practice issues. En M. L. Perlis, \& K. L. Lichstein (eds.), Treating Sleep Disorders: Principles and Practice of Behavioral Sleep Medicine (pp. 3-26). New York: Wiley.

Terman, M., Terman, J. S., \& Hall, M. (2000). Light therapy. En M. Kryger, T. Roth, \& W. Dement (eds.), Principles and practice of sleep medicine (3rd ed.) (pp. 1258-1274). Philadelphia: W.B. Saunders.

Weaver, T. E. (2001) Outcome measurement in sleep medicine practice and research. Part 1: assessment of symptoms, subjective and objective daytime sleepiness, health-related quality of life and functional status. Sleep Medicine Review, 5, 103-128.

Webb, W. B. (1988). An objective behavioral model of sleep. Journal Sleep, 11, 488-496.

Zadra A. L, \& Pihl, R. O. (1997) Lucid dreaming as a treatment for recurrent nightmares. Psychotherapy \& Psychosomatic, $66,50-55$. 